zierter Lehr- und Lernziele gesehen und eingesetzt werden, sondern die impliziten Lernziele könnten auch deutlicher formuliert und reflektiert werden.

Und auch im Zusammenhang mit der Darstellung juristischer Berufspraxis könnten die Qualitäten einer Universität differenziert genutzt werden. Die Aufgabe von "Wissenschaft « könnte in diesem Zusammenhang darin bestehen, die jeweils aktuelle anwaltliche Berufspraxis nicht nur zu reproduzieren, sondern sie auch theoretisch zu reflektieren und eine "Theorie der Praxis« zu bieten ${ }^{35}$. Schließlich will auch die aktuelle Debatte um alternative Konfliktlösung und das Harvard-Modell des Verhandlungsmanagements die tatsächliche Berufspraxis in Deutschland nicht beschreiben, sondern verändern ${ }^{36}$. Eine solche wissenschaftliche Behandlung von Konfliktlösungsmechanismen müßte interdisziplinär sein und mit Erkenntnissen der Organisationswissenschaften und Psychologie, aber auch der Sozialwissenschaften arbeiten. Hier wäre möglicherweise ein Ansatzpunkt für ein neues Verständnis von beispielsweise $\$ I Abs. 3 Satz 3 HambJAO: »Der Jurist soll lernen, soziale Hintergründe und Folgen rechtlicher Regelungen zu erkennen und diese Erkenntnisse ebenso wie die Ergebnisse anderer Wissenschaften kritisch würdigend in seine Entscheidung einzubeziehen. «37

\title{
Thomas Groß \\ Europäische Grundrechte als Vorgaben für das Einwanderungs- und Asylrecht
}

\section{Einleitung}

Einwanderung und Asyl sind zwei Politikfelder, die in der jüngeren Diskussion häufig miteinander verknüpft werden. Sie werden im geltenden deutschen und europäischen Recht aber sehr unterschiedlich behandelt. ${ }^{1}$ Hinter der rechtlichen Differenzierung stehen prinzipiell unterschiedliche Konzeptionen, die z. B. dadurch ausgedrückt werden, daß Einwanderung vorrangig aus ökonomischen Zwecken erlaubt werde, während das Asylrecht humanitären Zwecken diene. ${ }^{2}$ Einwanderung wird deshalb als Gebiet angesehen, in dem das Gastland nach politischem Ermessen entscheidet, welche und wie viele Personen es entsprechend der wirtschaftlichen Nützlichkeit einreisen läßt. Das Asylrecht ist dagegen nach Art. I6a GG ein Grundrecht und sollte folglich unabhängig von tagespolitischen Entscheidungen sein. Nichts desto trotz zeigt ein realistischer Blick auf das komplexe Phänomen der globalen Migrationsbewegungen, daß es offensichtlich Verbindungen zwischen beiden Aspekten gibt. ${ }^{3}$ Ein Flüchtling, der seit einigen Jahren in einem anderen Land

35 So der Titel der Lehrveranstaltung an der juristischen Fakultät in Hannover während der "Reformphase« (Kühling (Fn. 3I), S. 138).

36 Harald Koch (Fn. 3), 327; vgl. Gottwald (Fn, I6), S. 22 f;; Haft, BB-Beilageid/1998, S. I 8; siehe auch den Leserbrief von Harald Wozniewski, NJW 29/2000, S. XXV, der die oft gehörte Behauptung von AnwältInnen, „Wir betreiben doch sowieso schon Mediation«, zu Recht hinterfragt.

37 Siehe auch die Forderung von Jeand'Heur (Fn. 34), S. 428, angehende JuristInnen müßten nicht zuletzt einen verantwortlichen Umgang mit der Macht, über die sie vcrfügen könnten, lernen.

I Noll/Vested-Hansen, in: Alston/Bustelo/Heenan (Hrsg.), The EU and Human Rights, 1999, S. 359 ff., 363 ; Harvey, Public Law (PL) 1999, 23 ff., 24 ; Röben, in: Grabitz/Hilf (Hrsg.), Das Recht der Europäischen Union, Art. 63 EGV Rn. 33.

2 Schoch, DVBI. 1992, 525 ff., 536.

$3 \mathrm{Vgl}$. auch - allerdings unter negativen Vorzeichen - Pfaff, KJ 2000, $147 \mathrm{ff}$. 
lebt, kann ein Einwanderer werden. Ein Ausländer, der nur für einige Jahre bleiben wollte, kann zum Flüchtling werden, wenn sich die politische Situation in seinem Heimatstaat ändert, oder zum Einwanderer, wenn er im Gastland eine Familie gründet. Deshalb ist ein differenzierterer, integrierter Ansatz notwendig. Dies hat auch die englische Regierung in dem 1998 veröffentlichten Weißbuch »Fairer, Faster and Firmer - a Modern Approach to Immigration and Asylum « anerkannt. ${ }^{4}$

Die folgende Untersuchung soll sich dieser Problematik aus der speziellen Perspektive des europäischen Rechts nähern, das bereits heute die Basis für eine solche differenzierte Herangehensweise enthält. Ausgangspunkt sind dabei die in der Europäischen Menschenrechtskonvention und im Gemeinschaftsrecht enthaltenen Grundrechte. Sie beschränken inzwischen in nicht unerheblicher Weise die Freiheit des nationalen Gesetzgebers, bestimmte Rechte für Ausländer oder Flüchtlinge vorzusehen oder vorzuenthalten. Die wachsende Bedeutung dieser supranationalen Perspektive, die oft nur unter dem Gesichtspunkt zusätzlicher Beschränkungen der in Deutschland geltenden rechtlichen Situation wahrgenommen wird', sollte von nationalen Politikern, aber auch von Juristen ernster genommen werden.

Das wichtigste europäische Instrument des Menschenrechtsschutzes, die Europäische Menschenrechtskonvention, enthält mehrere Vorschriften, die für den hier untersuchten Fragekreis von Bedeutung sind. Von zentraler Bedeutung sind Art. 3, der Schutz vor Folter oder unmenschlicher oder erniedrigender Strafe oder Behandlung, und Art. 8, der Schutz des Familienlebens. Beide Garantien sind Gegenstand einer ausführlichen Rechtsprechung des Europäischen Gerichtshofs für Menschenrechte in Straßburg.

Neben diesem traditionellen internationalen Vertrag hat das Recht der Europäischen Gemeinschaft im letzten Jahrzehnt großen Einfluß auf das Einwanderungs- und Asylrecht gewonnen. Der Amsterdamer Vertrag hat der Gemeinschaft in Art. 6I, $6_{3}$ EG eine allgemeine Rechtsetzungskompetenz in diesem Bereich übertragen. Zwar ist das Sekundärrecht bisher noch wenig entwickelt. Auf der Grundlage der Vorgängervorschrift des Art. K.3 EUV in der Maastricht-Version ist nur ein Gemeinsamer Standpunkt angenommen worden, der zudem die nationalen Stellen nicht bindet. ${ }^{6}$ Die Kommission hat jedoch die Absicht, die Rechtsetzung voranzutreiben. Ein erster Vorschlag für eine Richtlinie über Minimalstandards für mitgliedsstaatliche Verfahren zur Anerkennung und Entziehung des Flüchtlingsstatus liegt inzwischen vor. ${ }^{7}$ Solange allerdings die Einstimmigkeit der Beschlußfassung, auf die die Bundesregierung besonderen Wert legt ${ }^{8}$, erhalten bleibt, dürften rasche Fortschritte kaum zu erwarten sein. ${ }^{9}$

Auch die auf dem Gipfel von Nizza proklamierte Charta der Grundrechte der Europäischen Union ${ }^{10}$ enthält einschlägige Vorschriften. Auch wenn sie zur Zeit noch kein geltendes Recht ist, ist doch anzunehmen, daß sie die Rechtsprechung bei der Anwendung der bestehenden Grundrechtsvorschriften beeinflussen wird. ${ }^{1}$ Drei Artikel betreffen insbesondere die Bereiche der Einwanderung und des Asylrechts. Art. 7 schützt das Familienleben mit der selben Formulierung wie Art. 8 EMRK. Art. 18 gewährleistet das Recht auf Asyl nach Maßgabe der Genfer Flüchtlingskon-

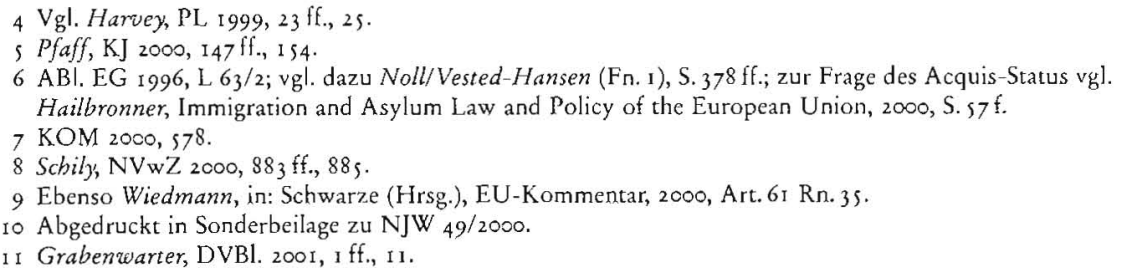


vention. Art. 19 verbietet Kollektivausweisungen sowie eine Abschiebung, Ausweisung oder Auslieferung an einen Staat, in dem das ernsthafte Risiko der Todesstrafe, der Folter oder einer anderen unmenschlichen oder erniedrigenden Strafe oder Behandlung besteht.

Im folgenden können nicht alle Aspekte der Problematik behandelt werden. Vielmehr soll der menschenrechtliche Ansatz dadurch deutlich gemacht werden, daß die Perspektive der betroffenen Menschen zum Ausgangspunkt genommen wird, um die rechtlichen Probleme zu strukturieren. Da die politische Debatte weitgehend auf Kontrolle und Beschränkung konzentriert ist, ist dies eine unübliche Herangehensweise. Sie erweist sich aber als nützlich, um den Einfluß der internationalen Verpflichtungen deutlich zu machen. Dabei spielen vier Hauptaspekte eine Rolle, die mehr oder weniger parallel zu einer individuellen Chronologie von Migration zu sehen sind:

Die erste zentrale Frage ist, ob es ein Recht zur Einreise gibt. Wird der Zutritt in das gewünschte Gastland verweigert, erübrigen sich alle weiteren Rechtsfragen. Deshalb sind die Regelungen über Grenzkontrollen und Visa von entscheidender Bedeutung für das Einwanderungs- und Asylrecht. Für alle diejenigen, die erfolgreich eingereist sind (legal oder illegal), einschließlich derjenigen, die in einem Staat geboren sind, ohne daß sie dessen Staatsangehörigkeit haben, stellt sich als weitere Frage mit mehr oder weniger der gleichen Bedeutung, ob sie ein Bleiberecht haben. Da die Abschiebung ein zentrales Instrument des Ausländerrechts ist, ist ihre Verhinderung ein vitales Interesse aller betroffenen Personen. Viele gewaltsame Übergriffe nicht nur in Deutschland, sondern mehr oder weniger überall in Europa, haben in den letzten Jahren deutlich gemacht, daß Einreise- und Bleiberecht nicht ausreichend sind, um einen effektiven Schutz von Einwanderern und Flüchtlingen zu gewährleisten. Rassistische und fremdenfeindliche Angriffe, die in den letzten ro Jahren in Deutschland den Tod von mehreren Dutzend Personen verursacht haben, aber auch subtilere Formen der Diskriminierung werfen die dritte Frage auf, ob Ausländer ein Recht auf staatlichen Schutz haben. Der vierte Aspekt des Themas geht über das Recht, in einem anderen Land friedlich leben zu können, hinaus und betrifft die nächsten Schritte einer Integration in die Gesellschaft durch die Gewährung sozialer und politischer Rechte. Es stellen sich Fragen wie die nach der sozialen Absicherung und nach einem Recht auf Mitwirkung an politischen Entscheidungsprozessen ${ }^{12}$. Da in einer Demokratie das Wahlrecht das wichtigste Instrument zur Verteidigung der Interessen ist, geht es vor allem um seine Ausweitung auf Einwanderer.

\section{Das Recht zur Einreise}

Die Kontrolle der Staatsgrenzen wird traditionell als ein Kernelement der Souveränität angesehen. ${ }^{13}$ Nichts desto trotz genießen die Staaten heute keine unbegrenzte Ermessensfreiheit mehr bei der Entscheidung, wen sie einreisen lassen.

\section{Die Freizügigkeit der Unionsbürger}

Art. 8 EG, der durch den Amsterdamer Vertrag eingefügt wurde, garantiert jedem Unionsbürger das Recht, sich im Hoheitsgebiet der Mitgliedstaaten frei zu bewegen und aufzuhalten. Die Unionsbürgerschaft ist nach Art. 17 Abs. 1 S. 2 EG akzessorisch

\footnotetext{
12 Dieser Zusammenhang wird thematisiert z. B. von Bryde, in: Weick (Hrsg.), National and European Law on the Threshold to the Single Market, I993, S. I $59 \mathrm{ff}$, I64 f; Wollenschläger, ZAR 1999, $256 \mathrm{ff}, 257$; ausführlich auch Wallrabenstein, Das Verfassungsrecht der Staatsangehörigkeit, 1999, S. 9off. 13 Noll/Vested-Hansen (Fn. I), S. 36 .
} 
zur Staatsangehörigkeit eines Mitgliedsstaates. Damit sind die bisher auf »Marktbürger « beschränkten Rechte der Freizügigkeit der Arbeitnehmer (Art. 39 EG) und des Rechts auf freie Niederlassung (Art. 43 EG) verallgemeinert worden. ${ }^{14}$ Deshalb stellt sich die Frage, ob es überhaupt noch sinnvoll ist, Angehörige anderer Mitgliedsstaaten der Europäischen Union als »Einwanderer « bzw. »Ausländer « zu bezeichnen. In einem bestimmten Umfang umfaßt die Freizügigkeit auch Familienangehörige von Unionsbürgern, die Angehörige von Drittstaaten sind. "

Art. 8 EG verweist in bezug auf die Begrenzungen der Freizügigkeit auf die aus Gründen der öffentlichen Ordnung, Sicherheit und Gesundheit gerechtfertigten Beschränkungen nach Art. 39 A bs. 3 , 46 EG, sowie das entsprechende Sekundärrecht. Diese Bestimmungen sind allerdings vom Europäischen Gerichtshof sehr restriktiv interpretiert worden. ${ }^{16}$ Deshalb gilt das Recht der Unionsbürger, in einem anderen Mitgliedsstaat der Union ihrer Wahl zu leben, fast unbeschränkt.

\section{Das Asylrecht}

Art. I6a Abs. I GG beinhaltet ein Recht auf Einreise, um Asyl zu beantragen. ${ }^{17}$ Das Bundesverfassungsgericht hat allerdings auch entschieden, daß es zulässig ist, asylsuchenden Ausländern die Verpflichtung aufzuerlegen, vor der Einreise in das Bundesgebiet eine Aufenthaltserlaubnis in der Form des Sichtvermerks einzuholen, sofern dem Betroffenen eine Erfüllung dieser Verpflichtungen möglich und zumutbar ist. ${ }^{18}$ Eine Bestimmung der Fälle, in denen die Zumutbarkeit zu bejahen ist, ist allerdings schwierig, da die Situation eines Flüchtlings es oft ausschließt, ordnungsgemäße bürokratische Verfahrensweisen einzuhalten. Das Erfordernis eines Visums ist mittlerweile vielmehr ein Hauptinstrument der Einreise-Verhinderungspolitik der europäischen Länder geworden. ${ }^{19}$

Eine andere wichtige Beschränkung enthält der Ausschluß des Einreiserechtes, soweit der Flüchtling über einen sicheren Drittstaat einreist. Die entsprechende Regelung in $\mathbb{1} 8$ Abs. 2 Nr. I AsylVfG beruht auf Art. I6a Abs. 2 GG und ist vom Bundesverfassungsgericht gebilligt worden. ${ }^{20} \mathrm{Da}$ alle Nachbarstaaten der Bundesrepublik zu sicheren Drittstaaten erklärt worden sind, ist das Flugzeug inzwischen der einzige Weg, um Deutschland legal als Asylbewerber zu betreten. Die Beförderungsunternehmer unterliegen allerdings nach $\$ 74$ AuslG einer Pflicht zur Kontrolle der Paß- und Visumspflichten, wozu sich die Bundesrepublik durch Art. 26 des Schengener Durchführungsabkommens von 1990 verpflichtet hat. Dieses System garantiert einen fast perfekten »Schutz " gegen die legale Einreise von Flüchtlingen.

Die Europäische Menschenrechtskonvention enthält kein Recht auf Asyl. ${ }^{21}$ Initiativen zu seiner Einführung durch ein Zusatzprotokoll sind nicht erfolgreich gewesen. Die Konvention hat deshalb nur indirekte Relevanz für Flüchtlinge.

Der neue Titel IV des EG-Vertrages verwendet in Art. 6I und 63 den Begriff »Asyl «,

I4 Oppermann, Europarecht, 2. Aufl., 1999, S. 653; Reich, Bürgerrechte in der Europäischen Union, 1999, S. I 8 ff., I 98 f.; restriktiv Craig/de Bürca, EU Law, 2. Aufl., 1998, S. 720.

is Dazu näher Hailbronner (Fn. 6), S. $176 \mathrm{ff}$

$16 \mathrm{Vgl}$. Craig/de Bürca (Fn. 14), S. $786 \mathrm{ff}$.

17 BVerfG NVwZ 1992, 973; Lübbe-Wolff, in: Drcier (Hrsg.), Grundgesetz, Bd. I, 1996, Art. 16а Rn. 54; Schnapp, in: v. Münch/Kunig (Hrsg.), Grundgesetz-Kommentar, Bd. 1, s. Aufl., 2000, Art. ía Rn.3; Waldstein, Das Asylgrundrecht im europäischen Kontext, 1993, S. 53.

I 8 BVerfG NVwZ 1987, 1068; s. a. Waldstein (Fn. 17), S. 47 ff.

I9 Noll/Vested-Hansen (Fn. I), S. $382 \mathrm{f}$.

20 BVerfGE 94, $49 \mathrm{ff}$.

2I BVerwGE 99, 331, 334; Hailbronner, ZAR 1995, 3 ff., I2; Kimminich, in: Dolzer/Vogel (Hrsg.), Bonner Kommentar zum Grundgesetz, Art. 16 Rn. $133 \mathrm{ff}$. 
um eine der Gemeinschaftskompetenzen im Rahmen des schrittweisen Aufbaus eines Raumes der Freiheit, der Sicherheit und des Rechts zu beschreiben. Nach dem Wortlaut der Vorschriften ist nicht klar, ob es sich ausschließlich um eine Kompetenzübertragung handelt oder ob die Regelung auch eine materielle Bedeutung hat, d. h. bindende Vorgaben für das Sekundärrecht enthält. Eine Direktwirkung dieser Artikel ist abgelehnt worden, weil die Mitgliedsstaaten ein Ermessen bei der Durchführung hätten und eine Übergangsperiode vorgesehen sei. ${ }^{22}$ Allerdings gibt es Parallelen zum Recht auf gleiches Entgelt für Männer und Frauen nach Art. I 4I EG (ex I I9 EWG), dem eine Direktwirkung vom Luxemburger Gericht zugestanden wurde, obwohl die Textbasis im Vertrag ähnlich ungewiß ist. ${ }^{23}$ Die Antwort auf die Frage, ob der EGVertrag eine implizite Anerkennung eines individuellen Asylrechts enthält, sowie nach dem Umfang eines entsprechenden Rechts, kann indes nur auf der Grundlage einer tiefergehenden, vergleichenden Untersuchung des nationalen Asylrechts und des Regelungsumfeldes gegeben werden. Sie muß hier offen bleiben.

Art. I 8 der Charta der Grundrechte der Europäischen Union garantiert explizit ein Recht auf Asyl. Der Verweis auf die Genfer Flüchtlingskonvention stellt eine Parallele zu Art. $6_{3}$ EG dar, der ebenfalls auf diese, alle Mitgliedsstaaten bindende internationale Verpflichtung hinweist. Auch wenn die Konvention selbst kein individuelles Asylrecht garantiert ${ }^{24}$, kann daraus keine Relativierung der Eigenschaft als individuelles europäisches Grundrecht abgeleitet werden, da sich diese aus der Aufnahme in die Charta ergibt. ${ }^{25}$ Welche Einschränkungen des Asylrechts zulässig sind, ist auf der Grundlage des Art. 52 der Charta zu entwickeln.

\section{Der Schutz des Familienlebens}

Der Schutz des Familienlebens in Art. 8 EMRK enthält nach einem oft zitierten Urteil des Straßburger Gerichtshofs kein Recht auf Einreise für ausländische Staatsangehörige. ${ }^{26}$ Das Hauptargument des Gerichtshofes war, daß die Staaten ein Recht zur Kontrolle der Einreise von Ausländern haben, so daß sie keiner generellen Verpflichtung unterliegen, die Wahl einer Familie, wo sie sich niederlassen will, zu respektieren. Allerdings muß diese Aussage im Zusammenhang mit der konkreten Fallgestaltung gesehen werden. Sie kann nicht so verstanden werden, daß ein Recht auf Einreise von Familienangehörigen von Personen, die in einem europäischen Land leben, generell ausgeschlossen und nicht von Art. 8 EMRK geschützt ist. Eine solch harte Schlußfolgerung wäre nicht nur unverhältnismäßig, sie übersieht außerdem, daß das Land, in dem die anderen Familienmitglieder leben, die die Einreise begehren, auch ein Recht auf eine Kontrolle der Einreise hat. Wenn dieses Land ebenso streng wäre, würde die Familie ewig getrennt bleiben. Um diese nicht tolerierbare Konsequenz zu vermeiden, ist eine gerechte Abwägung zwischen den Interessen der betroffenen Individuen und des Staates zu verlangen. ${ }^{27}$ Daraus folgt kein Recht auf unbeschränkte Einreise, doch muß ein Staat, der Familienangehörigen eines Einwohners die Einreise verweigert, ausreichende Gründe für diese Entscheidung nennen, die gerichtlich überprüft werden können. Dies hat nunmehr auch der Europäische Gerichtshof für Menschenrechte anerkannt, der im konkreten Fall allerdings die Einreiseverwei-

\footnotetext{
22 Hailbronner (Fn. 6), S.69.

23 EuGHE 1976, 455 (Defrenne II); vgl. Langenfeld, Die Gleichbehandlung von Mann und Frau im Europäischen Gemeinschaftsrecht, 1990, S. $29 \mathrm{ff}$

24 Klos, ZAR 2000, 202 ff., 203; Waldstein (Fn. 17), S. 58.

25 So auch Grabenwarter, DVBI. 2001, I ff., 5: $"$ Grundrecht auf Asylk.

26 EGMR A-94 (Abdulaziz u.a.) = EuGRZ 1985,567 , Ziff. 67 f.; s. a. BVerfGE 76, i, 47

27 BVerwG NVwZ I 998, 748, 750; Frowein/Peukert, EMRK-Kommentar, 2. Aufl., I996, Art. 8 Rn. 26.
} 
gerung für einen alleinstehenden 35 -jährigen Algerier, der wegen Heroinhandels in Frankreich bestraft und ausgewiesen worden war, für gerechtfertigt hielt. ${ }^{28}$

\section{Das Bleiberecht}

Die Ausweisung bzw. Abschiebung von Ausländern, denen kein Bleiberecht zusteht, ist eine notwendige Ergänzung des Rechts zur Grenzkontrolle, um Einwanderung steuern zu können. Es unterliegt allerdings auch Beschränkungen, die sich aus den Menschenrechten ergeben.

\section{Der Schutz gegen Folter}

Art. 3 EMRK wird in einer gefestigten Rechtsprechung des Europäischen Gerichtshofs für Menschenrechte als Ausschluß jeder Auslieferung oder Abschiebung interpretiert, sofern es ein ernsthaftes individuelles Risiko der Folter oder einer anderen unmenschlichen oder erniedrigenden Strafe oder Behandlung im Land, in das die Person geschickt wird, gibt. ${ }^{29}$ Dieser Schutz wurde auch auf Personen ausgedehnt, die eine entsprechende Verfolgung befürchten, die nicht von staatlichen Stellen droht, einschließlich einer drohenden Gefahr durch die Unmöglichkeit angemessener medizinischer Behandlung einer AIDS-Erkrankung in einem späten Stadium..$^{30} \mathrm{Als}$ Begründung für diese weite Auslegung weist der Gerichtshof auf den »absoluten Charakter des Schutzes « durch Art. 3 EMRK hin.

Obwohl diese Fälle sicher auf außergewöhnlichen Umständen beruhen, wie auch in den Urteilen des Gerichtshofes betont worden ist, hat die generelle Linie der Auslegung von Art. 3 EMRK offenen Widerspruch des Bundesverwaltungsgerichts provoziert. ${ }^{31}$ Es besteht auf der traditionellen deutschen Interpretation, wonach Schutz ausschließlich dann gewährt wird, wenn die gefürchtete unmenschliche Behandlung absichtlich von staatlichen Stellen zugefügt wird. Dementsprechend würde eine Abschiebung in Staaten ohne effektive Staatsmacht, wie es damals in Somalia der Fall war, nicht durch Art. 3 EMRK erfaßt. Die Rebellion ist umso bemerkenswerter, als diese Begründung im Ergebnis nicht ausschlaggebend war, da humanitärer Schutz letztlich doch gewährt wurde. ${ }^{32} \mathrm{Im}$ Hintergrund steht offensichtlich die Befürchtung, daß Art. 3 EMRK zu einem "Ersatzasylrecht« werden könnte. ${ }^{33}$

Unabhängig von der Frage, ob die weite Auslegung eines Menschenrechts ein Grund für Befürchtungen ist, wirft die Argumentation des Bundesverwaltungsgerichts die Frage der Vereinbarkeit mit der Verbindlichkeit der Menschenrechtskonvention auf. Auch wenn Art. ${ }_{3}$ EMRK die Pflicht, sich nach den Entscheidungen des Gerichtshofes zu richten, auf die Parteien des jeweiligen Falles beschränkt, macht ein System zentralisierter Auslegung durch ein unabhängiges Gericht, wie es durch die Konvention eingerichtet wurde, nur Sinn, wenn dessen Auslegung von allgemeiner Bedeutung für alle Vertragsstaaten ist. ${ }^{34}$ Nicht überzeugend ist es auch, eine Pflicht des Straßburger Gerichtshofes anzunehmen, auf eine veränderte politische Praxis der Mitgliedsstaaten zu reagieren. ${ }^{35}$ Die Rechtsprechung des Gerichtshofes ist erst we-

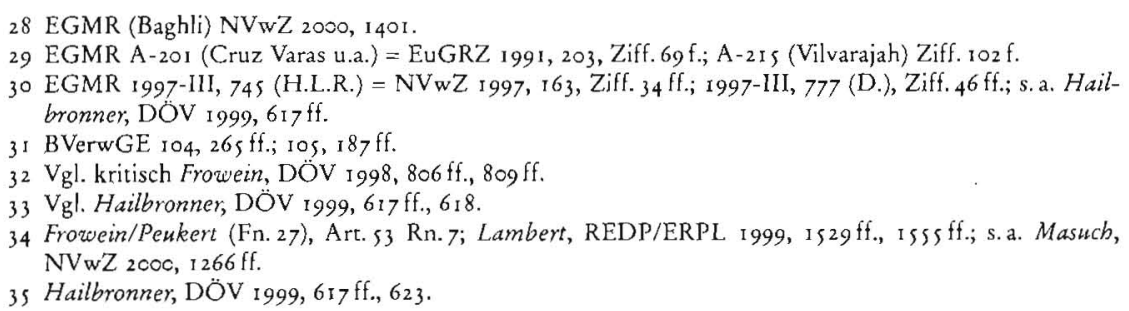


nige Jahre alt und beruht auf einer gründlichen Untersuchung der Fakten und des rechtlichen Hintergrunds.

Inzwischen gab es eine bemerkenswerte indirekte Korrektur des Bundesverwaltungsgerichtes durch das Bundesverfassungsgericht, das in einem vergleichbaren Fall, der Afghanistan betraf, entschieden hat, daß das Erfordernis der Verantwortlichkeit staatlicher Stellen nicht zu eng interpretiert werden darf. ${ }^{36}$ Es ist anzunehmen, daß dies auch eine Reaktion auf ein Urteil des englischen Court of Appeals war, der Deutschland den Status eines sicheren Drittstaates abgesprochen hat, weil es den Schutz von Flüchtlingen in solchen Fällen ablehne. ${ }^{37}$

Im Kern geht es um die politische Frage, wie die europäischen Staaten ihre Verantwortung für den Schutz der Menschenrechte interpretieren. Es ist kaum nachvollziehbar, eine globale Verpflichtung zum Schutz der Menschenrechte als eines der Grundprinzipien der Außenpolitik zu proklamieren, und sogar einen Krieg gegen Jugoslawien zu beginnen, um eine Minderheit zu schützen, wenn nicht gleichzeitig in der Innenpolitik die sich aus bindenden internationalen Verträgen ergebenden Pflichten zum effektiven Schutz aller Personen, die hier leben, erfüllt werden. ${ }^{38}$

\section{Der Schutz des Familienlebens}

Art. 8 EMRK umfaßt keinen absoluten Schutz von Familienmitgliedern vor Abschiebung, sofern die Voraussetzungen des nationalen Ausländerrechts für einen Aufenthalt nicht erfüllt sind. Allerdings darf eine Familie nur dann getrennt werden, wenn diese Maßnahme nach Art. 8 Abs. 2 EMRK gerechtfertigt ist. Deshalb ist es immer erforderlich zu untersuchen, ob die Abschiebung in einer demokratischen Gesellschaft notwendig ist, d.h. eine Abwägung zwischen den individuellen und den staatlichen Interessen vorzunehmen. ${ }^{39}$ Der wichtigste Faktor ist dabei die Intensität der Beziehung der betroffenen Person zum Gastland im Verhältnis zum Herkunftsland. Es ist deshalb zweifelhaft, ob es als Rechtfertigung für die Abschiebung eines vierzehnjährigen türkischen Jungen, der mehrfach straffällig geworden war, ausreicht, daß er die türkische Sprache beherrscht, um ihn ohne seine Eltern in die Türkei zu schicken..$^{40}$

\section{Das Asylrecht}

Ein provisorisches Bleiberecht während der Prüfung des Antrags eines Asylbewerbers ergibt sich unmittelbar aus Art. 16a Abs. I GG. ${ }^{41}$ Eine vorzeitige Abschiebung des Antragstellers in das Land, in dem er Verfolgung befürchtet, wäre mit einem Asylanspruch unvereinbar. Dies folgt auch aus der Pflicht des Non-Refoulement nach Art. 33 der Genfer Flüchtlingskonvention. Das vorläufige Bleiberecht ist deshalb auch durch Nr. 2 und 17 der EU-Ratsentscheidung über Minimalstandards für Asylverfahren gewährleistet, die unter der Kompetenz von Art. K. I des EU-Vertrages in der Maastricht-Version angenommen wurde, allerdings ohne für die Mitgliedstaaten verbindlich zu sein..$^{42}$

Auch hier gibt es allerdings eine Ausnahme für den Fall eines offensichtlich unbe-

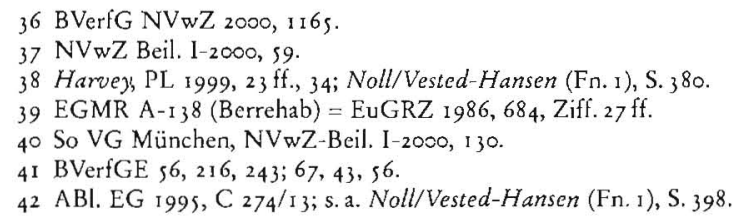


gründeten Antrags. Dic Möglichkeit einer Abschiebung, während ein Rechtsbehelf noch anhängig ist, ist durch Art. I6a GG gedeckt. ${ }^{43}$ Eine entsprechende Regelung enthält auch Nr. 2 I der genannten EU-Ratsentscheidung.

\section{Das Recht auf Schutz}

In modernen demokratischen Staaten, die sich zum Schutz der Grundrechte verpflichtet haben, ist der Staat nicht die einzige mögliche Gefahr für die Freiheit der Einwohner. Auch gewaltsame Attacken oder andere Formen der Diskriminierung durch Privatpersonen können gravierende Beschränkungen der freien Ausübung individueller Rechte bewirken. Deshalb ist der Schutz gegen Rassismus ebenfalls ein Menschenrechtsproblem.

\section{Der Schutz gegen Folter}

Der Europäische Gerichtshof für Menschenrechte hat in verschiedenen Fällen anerkannt, daß die Staaten verpflichtet sind, Grundrechte gegen Verletzungen durch Privatpersonen zu schützen, z. B. hat er den Schutz von Behinderten vor Vergewaltigung ${ }^{44}$ verlangt oder den Schutz vor Luftverschmutzung, die die Gesundheit von Nachbarn schädigt. ${ }^{45}$ Es erscheint als unproblematisch, diese Begründung auch auf das Recht auf Schutz vor Folter, unmenschlicher oder erniedrigender Behandlung, aber auch auf den Schutz des Rechts auf Leben (Art. 2 EMRK), zu übertragen und eine Pflicht des Staates zum Schutz vor entsprechenden Angriffen durch private Dritte zu begründen.

Auch hier stellt sich allerdings das generelle Problem bei Schutzpflichten, die ein positives Handeln staatlicher Stellen verlangen. Diese hängen nämlich von den Umständen und den verfügbaren Mitteln der Polizei oder anderer Behörden ab. Deshalb wird durchgängig anerkannt, daß es ein gewisses Ermessen in bezug auf die Art und Weise der Erfüllung der Schutzpflicht gibt. ${ }^{4^{6}}$ Ohne Zweifel ist es nicht möglich, allen Ausländern und Flüchtlingen jederzeit einen effektiven Schutz zu garantieren. Es ist aber andererseits ebenso klar, daß in den Fällen, in denen es in einem bestimmten Gebiet oder gegenüber einer bestimmten Gruppe immer wieder zu rassistischen oder gewalttätigen fremdenfeindlichen Angriffen kommt, die Polizei eine Handlungspflicht hat und versuchen muß, weitere Attacken zu unterbinden.

\section{Der Schutz gegen Diskriminierung}

Auch im Recht der Europäischen Union beginnt sich eine Doktrin der Schutzpflichten zu entwickeln. Der Europäische Gerichtshof hat eine Vertragsverletzung anerkannt, als der freie Handel im gemeinsamen Markt durch massive gewalttätige Proteste von französischen Bauern, die spanische Lastwagen mit Lebensmitteltransporten zerstörten, ohne daß die Polizei effektiv eingriff, behindert wurde. ${ }^{47}$ Man könnte diese Begründung ohne weiteres auf einen Fall übertragen, in dem das Recht auf Freizügigkeit von Unionsbürgern ernsthaft durch rassistische Angriffe in einem bestimmten Mitgliedsstaat behindert würde. Dieser Staat würde gemeinschaftsrechtliche Verpflichtungen nicht erfüllen, die allerdings nur Unionsbürger schützen.

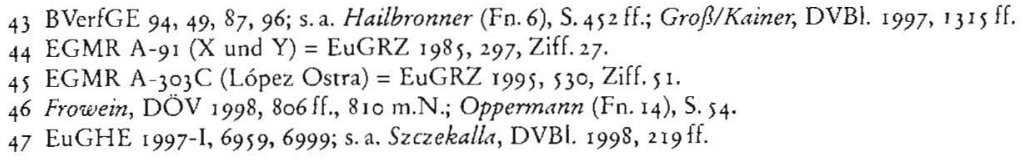


Auch Bürger von Drittstaaten werden durch Art. 29 EU erfaßt, der die Verhütung und Bekämpfung von Rassismus und Fremdenfeindlichkeit ausdrücklich als Ziel der Union eingeführt hat. ${ }^{48}$ Damit haben die Mitgliedstaaten anerkannt, daß es sich um ein allgemeines Phänomen handelt, das die nationale Verantwortlichkeit zur Aufrechterhaltung der öffentlichen Ordnung überschreitet. Im selben Zusammenhang steht Art. 3 EG, die Befugnis zum Erlaß von Antidiskriminierungsmaßnahmen, wodurch ebenfalls rassistische Diskriminierung bekämpft werden kann. ${ }^{49} \mathrm{Ob}$ sich aus diesen Kompetenzen auch individuelle Rechte ableiten lassen, falls diese Schutzpflichten nicht ausreichend erfüllt werden, ist eine offene Frage, die hier nicht weiterverfolgt werden kann.

\section{Das Recht auf Integration}

Der Aufenthalt bringt ökonomische und soziale Bedürfnisse mit sich, die ebenfalls menschenrechtliche Probleme aufwerfen. In diesem Bereich sind die Kataloge auf europäischer Ebene allerdings erheblich zurückhaltender. Da die Wanderungsbewegungen und die europäische Integration die Grenzen geöffnet haben und deshalb in allen europäischen Staaten eine nicht unerhebliche Zahl der Einwohner eine andere Staatsangehörigkeit haben, ist fast zwangsläufig eine Diskussion darüber aufgekommen, ob ihnen wirtschaftliche, soziale und politische Rechte gewährt werden sollen. Ohne Anspruch auf Vollständigkeit werden im folgenden der Zugang zum Arbeitsmarkt, zum Bildungssystem, zum Wahlrecht und zur Staatsangehörigkeit behandelt.

\section{Die Berufsfreibeit}

Die EMRK enthält keine Gewährleistung der Berufs- oder Gewerbefreiheit ${ }^{5 \circ}$, so daß sich auch die Frage einer Anwendung auf Ausländer nicht stellt. Dagegen sind die Freizügigkeit der Arbeitnehmer nach Art. ${ }_{9}$ EG und die Niederlassungsfreiheit nach Art. 43 EG Rechte, die alle Unionsbürger gleichstellen und zudem auf Studierende und Auszubildende ausgeweitet worden sind." Für Flüchtlinge und Einwanderer aus Staaten außerhalb der EU fehlt dagegen ein grundrechtlicher Schutz, der etwa gegen Arbeitsverbote oder andere Beschränkungen geltend gemacht werden könnte. Eventuell könnten sie sich allerdings im Anwendungsbereich des EG-Rechtes auf das Gemeinschaftsgrundrecht der Berufsfreiheit berufen, zu dessen Geltung für Drittstaatsangehörige sich der Luxemburger Gerichtshof bisher allerdings nicht geäußert hat. $^{52}$

\section{Das Recht auf Bildung}

Das Recht auf Bildung nach Art. 2 des I. ZP zur EMRK, das man als einziges soziales Grundrecht in der EMRK ansehen kann, gewährleistet einen diskriminierungsfreien Zugang zu Bildungseinrichtungen. ${ }^{33}$ Diese Vorschrift gilt nach Art. s 1. ZP i.V.m. Art. I EMRK unabhängig von der Staatsangehörigkeit. Folglich können auch Staats-

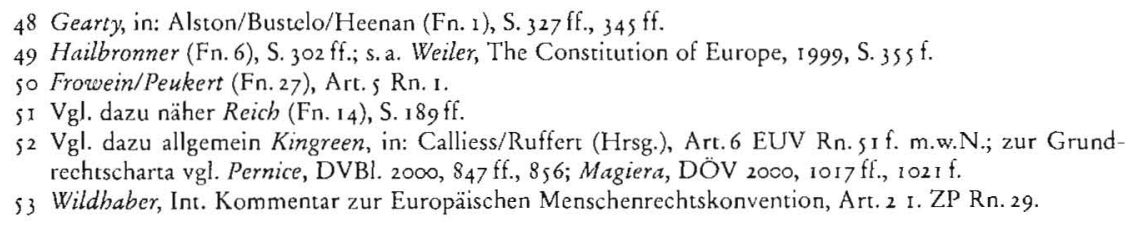


angehörige anderer Staaten im Land ihres Wohnsitzes nicht vom Schulbesuch ausgeschlossen werden. Strittig ist dabei, ob Kinder von Asylbewerbern wegen eines voraussichtlich kurzen Aufenthaltes ausgenommen werden können. ${ }^{54}$ Art. I4 der EU-Grundrechtscharta garantiert ebenfalls jeder Person das Recht auf Bildung und bezieht dabei ausdrücklich die Möglichkeit ein, unentgeltlich am Pflichtschulunterricht teilzunehmen.

\section{Das Wablrecht}

Das zentrale politische Recht in einer Demokratie ist das Recht, an Wahlen teilzunehmen. Seit dem Vertrag von Maastricht haben Unionsbürger das Wahlrecht bei Kommunalwahlen (Art. I9 Abs. I EG) und bei den Wahlen zum Europäischen Parlament (Art. 19 Abs. 2 EG). Nach der restriktiven Interpretation des Demokratieprinzips des Grundgesetzes durch das Bundesverfassungsgericht ist dies wohl schon die äußerste Grenze, Staatsangehörigen anderer Länder ein Wahlrecht zu gewähren. ${ }^{\text {s }}$ Die Angehörigen von Drittstaaten werden von der europäischen Gesetzgebung bisher nicht erfaßt.

Man könnte sich allerdings fragen, ob Einwohner mit der Staatsangehörigkeit eines anderen Staates nicht durch Art. 3 des ersten Zusatzprotokolles zur EMRK erfaßt werden. Es gewährleistet die freie Äußerung der Meinung des Volkes bei der Wahl der gesetzgebenden Organe, ohne seinem Wortlaut nach auf die Staatsangehörigkeit Bezug zu nehmen. Art. I EMRK, auf den in Art. s des ersten Zusatzprotokolles Bezug genommen wird, garantiert die Konventionsrechte allen der Jurisdiktion der Vertragsparteien unterstehenden Personen. Es handelt sich also um Menschenrechte, die unabhängig von der Staatsangehörigkeit gelten. Der Widerspruch, das Wahlrecht auf Staatsangehörige zu beschränken, ist offensichtlich bisher noch nicht erkannt worden. ${ }^{56}$ Die hier entwickelte systematische Auslegung kann wohl nur unter Hinweis auf die Entstehungsgeschichte widerlegt werden.

\section{Das Recht auf Einbürgerung}

Der letzte Schritt im rechtlichen Prozeß der Eingliederung, der allerdings nicht notwendig mit der sozialen Eingliederung verbunden ist, ist die Gewährung der Staatsangehörigkeit des Gastlandes. Dies wird offensichtlich nach wic vor als eine der Kernbefugnisse des Nationalstaates angesehen. Das Recht der Einbürgerung wird deshalb weder von der EMRK noch vom Recht der Europäischen Union erfaßt, wie auch ausdrücklich in der Erklärung Nr. 2 zum Amsterdamer Vertrag niedergelegt. ${ }^{57}$

\section{Fazit}

Es ist deutlich geworden, daß Einwanderung und Asyl einem Prozeß zunehmender Europäisierung unterliegen. Man kann auch von einer (graduellen) "Abdankung « des nationalen Ausländerrechts sprechen. ${ }^{58}$ Dieser Vorgang ist eine mehr oder weniger

54 Vgl. BayVGH DÖV 1997, 76; OVG Lüneburg, NVwZ-Beil. I-1999, 54; Langenfeld, Integration und kulturelle Identität zugewanderter Minderheiten in der Bundesrepublik Deurschland, 2001, S. 460 f. (zit. nach Typoskript).

ss BVerfGE 90, 37 ff.; zur Kritik vgl. nur die Beiträge in Redaktion Kritische Justiz. (Hrsg.), Demokratie und Grundgesetz, 2000.

56 Vgl. Wildhaber (Fn. 53), Art. 3 r. ZP Rn. 25, der die Beschränkung rechtfertigt, ohne Art. I EMRK zu erwähnen.

57 Hailbronner (Fn. 6), S. 9off.

s8 Schoch, DVBl. I 992,525 ff., 528. 
logische Konsequenz der Schaffung eines gemeinsamen Marktes, zumal die Integration inzwischen nicht mehr nur als rein ökonomisches Phänomen angesehen werden kann. ${ }^{59}$ In der Konsequenz des funktionalistischen Ansatzes hat die europäische Integration inzwischen ein Stadium erreicht, in dem alle Einwohner von der »immer engeren Union der Völker Europas« (Art. I EU) erfaßt werden. Wenn dadurch die internen Grenzen vollständig geöffnet werden, ist eine gemeinsame Regelung von Einwanderung und Asyl zwangsläufig notwendig. ${ }^{60}$

Es hat sich auch gezeigt, daß die Rechtsetzung im Bereich des Einwanderungs- und Asylrechts mehr und mehr durch europäische Grundrechte beeinflusst wird. Hierin spiegelt sich ein Prozeß der Differenzierung wider. Das europäische Recht reagiert auf die unterschiedlichen Lebenssituationen von Ausländern und Flüchtlingen und beseitigt damit zum 'Teil das ursprünglich anerkannte breite Ermessen der Nationalstaaten. Aus der vorhergehenden Analyse ergibt sich, daß es mindestens fünf verschiedene Klassen von Personen gibt, die durch unterschiedliche Regeln erfaßt werden:

- Die komplette Bandbreite der Grundrechte ist nur den Staatsangehörigen des jeweiligen Staates gewährleistet.

- Unionsbürger, d. h. Staatsangehörige eines anderen Mitgliedsstaates der Europäischen Union, genießen ein fast unbeschränktes Einreise- und Bleiberecht, aber nur einige Rechte auf Mitwirkung.

- Anerkannte Flüchtlinge, d. h. Personen, denen Asyl gewährt wurde, oder andere Flüchtlinge, die von Art. 3 EMRK geschützt werden, haben zumindest ein Bleiberecht.

- Einwanderer, die mit Familienangehörigen im Gastland leben, genießen einen graduellen Schutz durch Art. 8 EMRK.

- Alle anderen Ausländer werden von keinem der hier behandelten Rechte geschützt, ausgenommen das Recht auf Schutz vor Rassismus, das ein allgemeines Menschenrecht ist.

Diese Situation ist als neue »Apartheid « in Europa kritisiert worden. ${ }^{61}$ Es ist offensichtlich, daß es wesentliche Unterschiede im Rechtsstatus der verschiedenen Gruppen gibt. Auf der anderen Seite ist Staatsangehörigkeit ein traditionelles Element des Völkerrechts, das nicht primär geschaffen wurde, um zu diskriminieren, sondern um politische Einheiten zu formen. ${ }^{62}$ Inzwischen hat sich aber gezeigt, daß die globale Migration und die europäische Integration zu einer Relativierung dieser Form der Exklusion führen müssen. Die Anerkennung von Menschenrechten für Nicht-Staatsangehörige erweist sich aus dieser Perspektive als Fortschritt auf dem Weg zu Weltbürgerrechten. Die von Hannah Arendt beschriebene Situation, in der die Staatsangehörigkeit als $»$ Recht auf Rechte $\star^{63}$ eine Vorbedingung für einen effektiven Menschenrechtsschutz war, ist jedenfalls weitgehend überwunden worden. Das moderne internationale und insbesondere europäische Recht gewährt allen Menschen einen gewissen Schutz, der unabhängig von der Staatsangehörigkeit ist. Die Bindungswirkung der Menschenrechte erfaßt auch das Einwanderungs- und Asylrecht ${ }^{64}$ und erfüllt auch dort seinen wesentlichen Zweck, den demokratischen Entscheidungsprozessen Grenzen zu setzen. Gerade in diesem Bereich müssen die Regierungen

59 Anders Wiedmann (Fn. 9), Art.6I Rn. 4.

60 Weber, ZAR I993, i 1 ff., i7; Beckstein, ZAR 1999, I 1 ff.; Hailbronner, ZAR I995, 3 ff., I 1; a. A. Schily, NVwZ 2000,883 ff., 885

61 Balibar, lettre international Nr. $50(2000)$, S. 52

62 Zur Funktion der Nationalstaaten vgl. z. B. Weiler (Fn. 49), S. 447 ff;; Frankenberg, Die Verfassung der Republik, 1996, S.961.

63 Arendt, Elemente und Ursprünge totaler Herrschaft, Ausg. 1986, S. 462

64 Hailbronner (Fn. 6), S.88. 
öffentlich unter Druck gesetzt werden, daß sie die durch die Ratifikation internationaler Verträge und die Übertragung entsprechender Befugnisse auf die Europäische Union übernommenen bindenden Verpflichtungen umsetzen.

Von einer Bedrohung hoher deutscher Standards des Grundrechtsschutzes durch die Europäisierung kann also inzwischen keine Rede mehr sein. Bezeichnend ist, daß die Innenministerkonferenz eine kritische Stellungnahme zum Richtlinien-Entwurf der Kommission zum Flüchtlingsstatus ${ }^{65}$ abgegeben hat, da dieser viel zu großzügig sei ${ }^{66}$. Ähnliche Auseinandersetzungen gibt es beim Familiennachzug. Zur Erhaltung der Bremserrolle hat sich die Bundesregierung für die Beibehaltung des Einstimmigkeitsprinzips im Bereich des Einwanderungs- und Asylrechts eingesetzt, ${ }^{67}$ was ihr in der Konferenz von Nizza allerdings nur zum Teil gelungen ist. Der deutsche und der europäische Grundrechtsschutz haben sich in den hier behandelten Gebieten tendenziell konträr entwickelt. Folglich sollten auch die europäischen Arenen bei den Auseinandersetzungen über den Schutz der Rechte von Einwanderern und Flüchtlingen ernst genommen werden.

\section{Christoph Schminck-Gustavus Nemesis}

\section{Anmerkungen zum Urteil des Areopag zur Entschädigung griechischer Opfer von NS-Kriegsverbrechen (KJ 2000, S. 472 ff.)*}

Am I 3. April 2000 - knapp s6 Jahre nach dem Massaker von Dístomo, einer kleinen Ortschaft auf dem mittelgriechischen Festland - wurde im Athener Gerichtshaus eine historische Entscheidung verkündet. Die Vereinigten Senate des Areopag, also des obersten griechischen Gerichts, dessen zwanzig Richter auf hohem Podest unter einer großen Christus-Pantokrator-Ikone tagten, verwarfen mit großer Mehrheit die Revision der Bundesrepublik Deutschland gegen eine Entscheidung des Landgerichts Livadiá, mit der 269 Klägern aus Dístomo für die Ermordung ihrer Angehörigen und für die Niederbrennung ihrer Habe eine Entschädigung in Höhe von insgesamt 9,5 Milliarden Drachmen (umgerechnet 56 Millionen DM) zugesprochen worden war. Die Entscheidung des Areopag ist mit einer überraschend großen Mehrheit gefallen: sechzehn gegen vier Richter hatten sich über alle politischen Bedenken hinweggesetzt und den Klägern recht gegeben. So sah sich denn der Präsident des Areopag, der mit der Minderheit votiert hatte, ${ }^{1}$ genötigt, eine Entscheidung zu verkünden, die nicht nur für die deutsch-griechischen Beziehungen aufsehenerregend ist, sondern auch in der europäischen Rechtsgeschichte einmalig dasteht. ${ }^{2}$

Das Städtchen Dístomo liegt in der Nomarchie ${ }^{3}$ Böotien, unmittelbar neben dem

* Ich danke Herrn Prof. Argýris N. Sfountoúris (Athen/Zürich), einem Überjebenden der Tragödie von Dístomo, für zahlreiche Unterlagen und Hinweise zur ethischen Dimension des Themas.

I Vgl, die Berichte von Gerd Höhler, in: Frankfurter Rundschau vom I 4. 4. 2000, und von Ioánna Mándrou, in: To Vima yom 14.4 .2000 , S.A 17 .

2 Als einzige juristische Fachzeitschrift in der Bundesrepublik hat die $\mathrm{KJ}$ in Heft $3 / 2000, \mathrm{~S}_{4} 47_{2} \mathrm{ff}$., eine Übersetzung des Urteils in Auszügen abgedruckt.

3 Die den französischen Départements nachgebildeten griechischen Nomarchien entsprechen als Verwaltungseinheiten in etwa den deutschen Regierungsbezirken.
} 